Cent ans d'évolution des manuels de géographie pour la classe terminale en France : les profondes transformations du rapport aux savoirs scolaires en géographie

100 years of evolution of geography textbooks for year 12 students in France : deep transformations in the relationship to school knowledge in geography

Cien años de evolución de los libros de texto de geografía para alumnos de $5^{\circ}$ de secundaria en Francia : las profundas transformaciones de la relación con los saberes escolares en geografía

Daniel Niclot

Volume 35, numéro 2, 2009

Les manuels scolaires : réformes curriculaires, développement professionnel et apprentissages des élèves

URI : https://id.erudit.org/iderudit/038732ar

DOI : https://doi.org/10.7202/038732ar

Aller au sommaire du numéro

Éditeur(s)

Revue des sciences de l'éducation

ISSN

0318-479X (imprimé)

1705-0065 (numérique)

Découvrir la revue

Citer cet article

Niclot, D. (2009). Cent ans d'évolution des manuels de géographie pour la classe terminale en France : les profondes transformations du rapport aux savoirs scolaires en géographie. Revue des sciences de l'éducation, 35(2), 129-153.

https://doi.org/10.7202/038732ar
Résumé de l'article

Cette étude présente une analyse des manuels de géographie français de la classe terminale sur une période de cent ans ainsi que de leur évolution pédagogique. Elle s'appuie sur l'analyse de 21 ouvrages scolaires publiés entre 1905 et 2006. Elle vise à identifier les principaux modèles pédagogiques auxquels ils peuvent être référés. Les transformations des manuels sont mises en relation avec l'évolution du système éducatif français d'une part et, d'autre part, avec l'évolution du rapport aux savoirs scolaires en géographie des élèves et des enseignants. 


\section{Cent ans d'évolution des manuels de géographie pour la classe terminale en France : les profondes transformations du rapport aux savoirs scolaires en géographie}

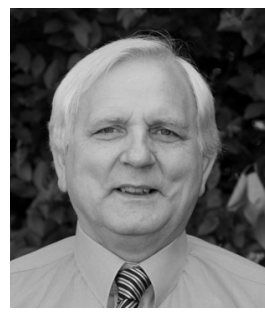

Daniel Niclot, maître de conférences Université de Reims Champagne-Ardenne

RÉSUMÉ - Cette étude présente une analyse des manuels de géographie français de la classe terminale sur une période de cent ans ainsi que de leur évolution pédagogique. Elle s'appuie sur l'analyse de 21 ouvrages scolaires publiés entre 1905 et 2006. Elle vise à identifier les principaux modèles pédagogiques auxquels ils peuvent être référés. Les transformations des manuels sont mises en relation avec l'évolution du système éducatif français d'une part et, d'autre part, avec l'évolution du rapport aux savoirs scolaires en géographie des élèves et des enseignants.

MOTS CLÉS • géographie, France, manuel scolaire, rapport au savoir, modèle pédagogique.

\section{Introduction et problématique}

Les critiques portées en France sur les manuels scolaires actuels, du primaire comme du secondaire, mettent en lumière le fait que les documents, à la qualité esthétique irréprochable, mais présentant peu d'intérêt didactique, prennent une place accrue, au détriment du cours, réduit à quelques lignes par chapitre dans certains ouvrages du primaire ou du collège. Les manuels ne sont plus comme jadis des livres de textes qui se lisent de manière linéaire. L'intertextualité s'est développée, renvoyant le lecteur à des documents divers, des activités, des définitions. Parallèlement, les exercices, les questionnements et les activités de tous types auraient pris une place exagérée, limitant l'autonomie pédagogique des enseignants. Certains le déplorent et y trouvent la cause d'une certaine désaffection de la part des élèves (Inspection générale de l'Éducation nationale, 1998; Le Fustec et Sivan, 2006).

Le propos développé ici est quelque peu différent. Il concerne d'abord les manuels français d'une discipline particulière, la géographie, et d'une classe spécifique, la 
terminale, qui accueille les élèves de 17 et 18 ans pour leur dernière année d'études secondaires. La classe terminale est sanctionnée par un examen: le baccalauréat, qui comporte une épreuve d'histoire et de géographie obligatoire. Sauf cas exceptionnel, le succès au baccalauréat est nécessaire pour accéder à l'enseignement supérieur.

Alors que les études portant sur les manuels scolaires se limitent souvent à ceux en usage au moment où elles sont réalisées, c'est une étude sur le long terme qui est présentée. En analysant 21 ouvrages scolaires publiés entre 1905 et 2006, notre objectif est de mieux comprendre les caractéristiques des manuels de la classe terminale d'aujourd'hui. L'étude qui identifie les principaux modèles pédagogiques auxquels ils peuvent être référés est l'occasion de s'interroger sur les transformations des manuels, qui sont mises en relation avec l'évolution du système éducatif français d'une part et, d'autre part, avec l'évolution du rapport aux savoirs scolaires en géographie des élèves et des enseignants.

Dans le présent article, à l'intérieur d'une première partie, le contexte théorique de cette étude sera précisé; puis, dans la deuxième partie, nous expliciterons la méthodologie d'analyse des manuels scolaires; enfin, les résultats des analyses menées sur les manuels scolaires seront présentés.

\section{Contexte théorique}

\subsection{La notion de système manuel}

Beaucoup de recherches menées sur les manuels scolaires (Chevalier, 2002; Desiré et Pagney, 1996; Freycinet-Deminjon, 1969; Lefort, 1992; Lucas, 2001; Mendibil, 1997; Sourp, 2002) ont privilégié les études de contenu. Elles se sont surtout intéressées au texte du cours analysé selon des perspectives qui se référent à la didactique de la discipline scolaire concernée. Elles se sont également centrées sur les méthodes d'apprentissage mises en œuvre par les manuels et sur les conceptions pédagogiques de leurs auteurs. Une autre approche très féconde a été celle de l'étude de l'idéologie véhiculée par les manuels scolaires. Les analyses présentées ici ne s'appuient pas sur des études de contenu menées selon une perspective didactique, épistémologique ou idéologique. Elles proposent une autre entrée pour accéder à une meilleure compréhension des manuels scolaires, qui ne se substitue pas aux approches citées précédemment, mais les complète.

La notion de système manuel (Niclot, 2001) et les compléments apportés (Lenoir, 2006) mettent en évidence la multiplicité des facteurs expliquant l'évolution de la production des manuels scolaires, objets didactiques mais aussi médias de communication sociale. Dans cette perspective, le manuel est envisagé comme un tout formé d'éléments interdépendants. Certains éléments sont formels et relèvent des techniques d'édition (de la Borderie, 1997), d'autres sont idéologiques, didactiques, pédagogiques. Le système manuel peut être considéré comme un système ouvert: [...] en relation permanente avec son environnement, il échange énergie, matière, informations utilisées dans le maintien de son organisation contre la dégradation qu'exerce le temps (de Rosnay, 1975, p. 32). 
L'environnement du système manuel est constitué par des métasystèmes avec lesquels les ouvrages scolaires échangent des flux d'informations conditionnant son évolution. Les principaux métasystèmes en connexion avec les manuels sont les savoirs savants (géographiques, didactiques, pédagogiques), le système scolaire et le système didactique, la société globale (qui fournit des informations sur les évolutions du monde et des sociétés), le système économique (qui conditionne la production des manuels scolaires dans ses aspects matériels), le système médiatique et l'idéologie dominante (Lenoir, 2006). Toutes les informations fournies par ces métasystèmes concourent à transformer les systèmes manuels en permanence, même si des effets de latence existent.

Le propre de l'approche systémique est de refuser toute hiérarchie entre les éléments qui composent le système. À la suite d'une identification des éléments constitutifs, on trouve une étude des interrelations entre les éléments qui vise à caractériser et à comprendre le fonctionnement du système. C'est dans cette perspective que s'inscrit cette étude de la structure formelle des manuels, notamment de l'évolution du nombre de pages consacrées à l'exposé du cours, aux documents, aux activités de nature diverse, autant d'éléments peu étudiés, voire ignorés, dans les études classiques. L'idée force de la démarche adoptée est que la répartition de ces différents éléments constitutifs n'est ni aléatoire ni le résultat de simples innovations techniques permettant, par exemple, de faire figurer plus de documents photographiques ou de généraliser l'usage de la typographie en couleur. Une telle étude ne s'attache pas à des aspects périphériques ou secondaires, elle repose sur l'hypothèse que l'étude de la structure des manuels permet de mettre en évidence les intentions pédagogiques et didactiques ou les conceptions qu'ont les auteurs de manuels des apprentissages des élèves. Notons que ces intentions ont été influencées en amont par l'évolution du système éducatif français qui a été considérable durant cette période.

\section{2 Évolution du système éducatif et transformation des manuels scolaires}

Au cours du siècle dernier, la société française a connu d'importantes mutations qui se sont répercutées sur l'enseignement en général et sur celui de la géographie en particulier et, par conséquent, sur les manuels scolaires. Pour comprendre les transformations des manuels scolaires français durant cette période d'un siècle, trois éléments de contexte sont présentés. Ils concernent la succession des programmes, les transformations de l'épreuve de géographie au baccalauréat et la diversification du public scolaire liée au mouvement de massification de l'enseignement secondaire français.

\subsubsection{Les changements de programmes}

Les changements de programmes sont un premier élément important à prendre en compte dans l'étude des manuels scolaires, dans la mesure où ils conditionnent non seulement les contenus, mais aussi la production de nouveaux ouvrages. Les 
programmes français sont constitués d'une liste exhaustive de sujets à étudier, complétés par des instructions officielles (qui deviendront à la fin des années 1980 des documents d'accompagnement). Ces derniers donnent aux enseignants, et aux auteurs de manuels scolaires, des conseils pour mettre en œuvre les programmes.

Tableau 1

La succession des programmes de géographie de la classe terminale de 1905 à 2001

\begin{tabular}{ll}
\hline $\begin{array}{l}\text { Date de } \\
\text { parution } \\
\text { du programme }\end{array}$ & Titre ou contenu du programme \\
\hline 1905 & Les principales puissances économiques du monde \\
1925 & Les principales puissances économiques du monde \\
1938 & Les principales puissances économiques du monde \\
1943 & Géographie générale (programme non appliqué) \\
1945 & Les principales puissances économiques du monde. Le partage des matières \\
1963 & premières \\
1969 & Les grands problèmes du monde contemporai. \\
1977 & Les grands problèmes du monde contemporain. Les principales puissances \\
1981 & économiques du monde \\
1987 & Les grands problèmes du monde contemporain. Les principales puissances \\
1995 & économiques du monde \\
2001 & Les grands problèmes mondiaux. Trois grandes puissances. États-Unis, URSS, \\
\hline
\end{tabular}

Comme l'indique le tableau 1, entre 1905 et 2001, 12 programmes de géographie se sont succédé. Les dates qui figurent sur le tableau ci-dessus sont celles de la publication officielle des programmes, mais il faut noter qu'un délai plus au moins long (jamais inférieur à un an) s'écoule entre la date de parution des programmes et leur mise en application. Il permet aux éditeurs de concevoir et de réaliser les manuels scolaires et aux enseignants de préparer leurs cours. À titre d'exemple, le programme de la classe terminale paru en 1987 est mis en application en septembre 1989, et les manuels conformes à ces nouveaux programmes paraissent en mai 1989.

C'est par une circulaire du 28 juillet 1905 qu'a été introduit un enseignement de la géographie en classe terminale; jusqu'à cette date, les cours de cette discipline s'arrêtaient en classe de première. Ce programme est centré sur l'étude des grandes puissances du monde, qui correspondent à huit États d'Europe et à leurs colonies, deux d'Amérique du Nord (les États-Unis et le Canada), deux d'Amérique du Sud (le Brésil et l'Argentine), deux d'Asie (le Japon et la Chine), auxquels il faut ajouter la Nouvelle-Zélande et l'Australie. En une trentaine d'heures, les principales puissances mondiales et leurs colonies, à l'exception de la France, doivent être étudiées, note Clerc (2002, p. 133). L'étude de chaque État obéit à la même logique. Elle commence par la description du milieu (relief, sol, climat), puis passe à l'inventaire des principales ressources minérales et agricoles, à la population et à la description 
des principales activités industrielles. Elle se termine par l'étude des grandes régions géographiques, dont le facteur d'unité est généralement naturel (relief, géologie, climat). La logique adoptée par les programmes est surtout économique, la puissance étant présentée comme une conséquence de l'abondance des ressources naturelles dont dispose le pays. Le contenu et la logique des différents programmes de la classe terminale restent les mêmes sur le fond jusqu'en 1987. Les seuls changements notables concernent des fluctuations du nombre de puissances au programme, qui oscille entre 10 et 20 durant la période. Les programmes et les compléments comportent peu d'indications pédagogiques. Le modèle implicite dominant est celui du cours magistral. Toutefois, à partir des programmes de 1938, la nécessité d'appuyer la réflexion des élèves sur l'étude de documents est évoquée.

Une rupture intervient donc avec le programme paru en 1987: il témoigne d'abord d'un intérêt tout particulier porté aux logiques économiques, démographiques et géopolitiques de l'espace mondial et notamment aux questions de développement. De nouveaux espaces d'étude apparaissent dans le programme, comme l'aire Pacifique; quant au nombre de puissances étudiées, il se limite désormais aux États-Unis, à l'URSS, à la Chine, au Japon, à l'Inde et au Brésil. Le plan traditionnel est très critiqué dans les compléments du programme de 1987. Il y est conseillé de problématiser l'étude des grandes puissances, d'abandonner la perspective exhaustive et de centrer les analyses uniquement sur les éléments qui expliquent la puissance. Ce programme vise à donner des clés de compréhension aux élèves sur le fonctionnement du monde contemporain à l'échelle planétaire. Désormais, la réflexion personnelle et la construction des savoirs par les élèves à partir de l'analyse de documents sont privilégiées. Les programmes qui paraissent en 1995 et en 2001 conservent cette perspective. Le dernier de ces programmes va encore plus loin dans le changement. Il abandonne la référence à la logique des États-puissances au profit de celle des espaces de la puissance. Par exemple, dans ce programme, ce ne sont plus les États-Unis seuls qui sont étudiés, mais un espace nord-américain qui comprend les États-Unis et le sud du Canada.

\subsubsection{La classe terminale et le baccalauréat}

Un second élément à prendre en compte dans l'étude des manuels scolaires de la classe terminale est la spécificité de cette classe dans le système éducatif français. Les études sont sanctionnées par un examen de fin d'études secondaire. Avec l'introduction d'un enseignement de géographie en classe de terminale en 1905, une épreuve écrite de géographie, commune avec celle d'histoire, a été instituée au baccalauréat. Le temps imparti pour les deux disciplines était de quatre heures durant lesquelles le candidat devait rédiger une dissertation d'histoire et une dissertation de géographie. La dissertation du baccalauréat portait alors sur un sujet de cours et réservait une large place à la restitution de savoirs mémorisés. En 1961, au nom de la lutte contre le surmenage scolaire, l'histoire et la géographie deviennent des épreuves uniquement orales, passées par une minorité d'élèves, ceux qui 
obtiennent entre 8 et 12 sur 20 aux épreuves écrites, parmi lesquelles ne figurent ni l'histoire ni la géographie. Puis, le retour de ces deux disciplines à l'écrit a été décidé en 1981. À une époque où les autorités politiques déplorent une perte de repères chez les jeunes générations, la réintroduction de l'histoire et de la géographie à l'écrit du baccalauréat est considérée comme un moyen de renforcer la formation civique des élèves. La nature de l'épreuve change, puisque les candidats ont le choix entre trois sujets de géographie, dont un commentaire de documents et deux dissertations. Les sujets ne font plus expressément référence à des questions de cours, mais nécessitent un effort de classement et d'organisation des connaissances. Tous les sujets de dissertation comportent l'obligation de réaliser un croquis de géographie. De nouvelles épreuves de géographie du baccalauréat sont définies pour la session de 1999 (Ministère de l'Éducation nationale, 1997). Si, comme auparavant, l'épreuve est toujours commune à l'histoire et à la géographie, un tirage au sort préalable détermine la place de chaque discipline, en première ou en deuxième partie. Les épreuves de la première partie sont plus longues et le candidat choisit son sujet parmi deux compositions à rédiger et une étude de documents. Si la géographie est en seconde partie, le candidat doit réaliser un croquis. Les nouvelles épreuves nécessitent la mise en œuvre de compétences clairement définies. Par exemple, pour le commentaire de document, le texte officiel prévoit que le candidat, après avoir présenté les documents, sélectionne, classe, confronte les informations et rédige une réponse argumentée à la problématique du sujet, limitée à environ 300 mots.

Outre l'évolution des programmes et des épreuves du baccalauréat, une troisième transformation, qui conditionne en partie les deux premières, s'est produite durant la période d'étude, celle du passage d'un enseignement secondaire destiné à une élite à un enseignement pour le plus grand nombre. Cette transformation est communément appelée massification de l'enseignement secondaire. À titre indicatif et pour bien comprendre l'ampleur du phénomène, d'après les chiffres ministériels de la Direction de l'évaluation de la prospective et de la performance (DEPP) du ministère français de l'Éducation nationale, il y avait 15000 candidats au baccalauréat en 1930, 81000 en 1960 et près de 300000 en 2005; ce dernier nombre concerne uniquement les baccalauréats généraux, ceux dont les manuels ont été étudiés, à l'exclusion des baccalauréats professionnels. Si l'augmentation du nombre d'élèves de l'enseignement secondaire commence dans les années 1960, la réforme de 1977 du ministre de l'Éducation nationale Haby va accélérer le mouvement avec la création du collège unique qui accueille tous les jeunes Français de 12 à 16 ans. La massification des effectifs des élèves de collège entraîne une forte augmentation des effectifs des élèves en lycée, quatre ans plus tard en 1981, une fois que les élèves ont accompli leur scolarité au collège. L'hétérogénéité du public lycéen s'accroît dans la mesure où il n'est plus majoritairement composé d'élèves issus de milieux culturellement favorisés qui comprennent les enjeux des savoirs et des disciplines scolaires. 


\section{Méthodologie}

\subsection{Choix des manuels étudiés}

L'étude des transformations des manuels scolaires sur une période de cent ans se trouve confrontée à la question de la sélection des manuels à étudier. L'analyse exhaustive de l'ensemble de la production parue durant cette période s'avère problématique parce que certains manuels sont difficiles à trouver, mais aussi en raison de la lourdeur des analyses à mener sur un grand nombre d'exemplaires. Ainsi, on peut estimer à plus de cent le nombre de manuels de géographie de terminale publiés en France entre 1905 et 2005.

La sélection pourrait porter sur les manuels les plus prescrits par les enseignants ou les plus vendus par les maisons d'édition, c'est-à-dire les manuels les plus utilisés dans les classes. Or, les éditeurs français se refusent à divulguer leurs chiffres de vente des manuels récents, et aucune statistique n'est publiée pour les manuels les plus anciens. La question de la sélection des manuels pourrait aussi être résolue par un choix aléatoire, mais celui-ci se heurte à une contrainte matérielle qui est celle de pouvoir disposer de l'ensemble de la production. Face à ces difficultés, nous avons procédé à une sélection qui repose sur une connaissance personnelle de l'ensemble de la production. Elle tient compte également des travaux de chercheurs universitaires qui ont travaillé sur ces manuels scolaires et qui en ont apprécié la diffusion.

Le fait que le choix de manuels scolaires étudiés ne repose pas sur des critères clairement déterminés compromet-il la validité de l'étude? Nous ne le pensons pas. Le concept de système manuel, référent sur lequel s'appuie notre étude, postule que la production de manuels scolaires obéit à des logiques spécifiques de l'époque où ils sont produits. Il apparaît qu'une étude qui tente de comprendre les aspects communs des manuels scolaires, et non les traits distinctifs de chacun, peut s'accommoder du fait que le choix des ouvrages étudiés ne résulte pas d'un échantillonnage permettant de déterminer les plus représentatifs.

L'objet de cette étude est de mettre en évidence les caractéristiques des manuels actuels en étudiant leur évolution sur une longue période. La perspective adoptée vise à identifier les caractéristiques générales des manuels de géographie les plus anciens puis, à partir du constat dressé, de mener des études plus détaillées sur les différentes transformations qu'ont connues les ouvrages depuis 25 ans. C'est ce qui explique que les ouvrages scolaires publiés depuis les années quatre-vingt soient bien plus nombreux dans notre corpus (15) que les ouvrages antérieurs (6) à cette période. 
Tableau 2

Listes des manuels étudiés

Numéro des

manuels étudiés Auteur(s), année d'édition, titre, éditeur

Manuel 1 Fallex, M. et Mairey, A. (1905). Les principales puissances du monde (moins la France). Paris, France: Delagrave.

Manuel 2 Fallex, M. et Mairey, A. (1922). Les principales puissances du monde (moins la France). Paris, France: Delagrave.

Manuel $3 \quad G a l l o u e ́ d e c$, L. et Maurette, F. (1929). Les grandes puissances du monde. Classes de philosophie et de mathématiques divisions A et B. Paris, France : Hachette.

Manuel 4 Fallex, M., Mairey, A. et Gibert, A. (1932). Les principales puissances du monde (moins la France). Classe de Philosophie. Paris, France: Delagrave.

Manuel 5 Prévôt, V. (1955). Géographie du monde contemporain. Classes terminales, classes préparatoires aux grandes écoles. Paris, France: Belin.

Manuel $6 \quad$ Prévôt, V. (1966). Géographie du monde contemporain. Classes terminales A, B, C, D. Paris, France : Belin.

Manuel 7 Froment, R. et Kienast, R. (1983). Géographie. Terminales. Paris, France: Delagrave.

Manuel 8 Baleste, M. (1987). Le monde d'aujourd'hui. Paris, France: Armand Colin.

Manuel 9 Frémont, A. (1989). Géographie. Terminales. Paris, France: Bordas.

Manuel 10 Gauthier, A. (1989). Géographie. Terminales. Paris, France: Bréal.

Manuel $11 \quad$ Hagnerelle, M. et Prévot, V. (1989). Le système monde en questions. Terminales A, $B, C, D$. Paris, France: Magnard.

Manuel 12 Knafou, R. (1989). Géographie. L'espace mondial. Paris, France: Belin.

Manuel 13 Martin, J. et Pernet, L. (1989). Géographie du temps présent. Classes de Terminales. Collection GHREG. Paris, France : Hachette.

Manuel 14 Pitte, J.-R. (1989). Géographie. Terminales. Paris, France: Nathan.

Manuel 15 Bouvet, C. (1998). Géographie terminales L, ES, S. L'Espace mondial. Paris, France: Hachette.

Manuel 16 Ciattoni, A. (1998). Géographie. Terminales. Nouveau programme. Paris, France: Hatier.

Manuel 17 Gauthier, A. (1998). L'espace mondial. Paris, France: Bréal.

Manuel $18 \quad$ Hagnerelle, M. (1998). L'espace mondial. Paris, France: Magnard.

Manuel 19 Pitte, J.-R. (1998). Géographie. L'espace mondial. Terminales. Paris, France: Nathan.

Manuel 20 Joyeux, A. (2004). Géographie. Terminales ES, L, S. Paris, France: Hachette.

Manuel 21 Ciattoni, A. (2004). Géographie. Terminales L,ES, S. Paris, France: Hatier.

Note: Les ouvrages sont classés selon leur année de parution. Conformément à un usage établi dans l'étude des manuels scolaires, seul le nom du directeur de collection est cité.

\subsection{Méthode d'analyse}

Les analyses menées consistent d'abord à identifier les différentes unités éditoriales contenues dans chaque manuel. Une unité éditoriale se définit comme un ensemble de pages homogènes quant à leur forme, leur contenu et leurs intentions pédagogiques. Pour l'ensemble des manuels scolaires de géographie, nous pouvons identifier quatre grands types d'unités éditoriales:

1) Les pages de savoirs propositionnels (on parle aussi dans les écrits de recherche de cours, de savoir déclaratif) constituent un premier type d'unité éditoriale. La notion de savoir propositionnel est ainsi définie par Delbos et Joron (1984, p. 98): 
[...] quand nous appelons "propositionnel" le savoir scolaire, nous attirons l'attention sur l'une de ses caractéristiques marquantes, qu'à défaut de pouvoir être théorique, il résume le savoir, sous forme de propositions logiquement connectées et qui se contentent d'énoncer des contenus.

2) Les pages documentaires constituent un second type d'unité éditoriale. Les documents sont très variés dans les manuels de géographie. Les plus fréquents sont les photos, les textes de nature diverse, les tableaux statistiques, les graphiques. Parmi ceux-ci, la carte géographique est le document le plus spécifique de la discipline.

3) Un troisième type d'unité éditoriale est constitué par des pages mixtes qui comportent des documents (cartes, croquis, photos, etc.) au sein du texte du cours.

4) Enfin, un quatrième type d'unité éditoriale regroupe non seulement des documents de nature diverse, dont des exercices de préparation au baccalauréat, des éléments de réflexion métacognitive, mais aussi des lexiques et des glossaires. Ces unités éditoriales ont pour objectif de susciter la réflexion et le travail personnel de l'élève, en l'aidant à mémoriser ou à mieux comprendre les savoirs développés dans le manuel, ou en lui proposant des exercices à réaliser. Nous appelons pages d'activités celles qui relèvent expressément d'une intention de mise en activité des élèves.

Pour chaque manuel, la méthodologie consiste à identifier les différentes unités éditoriales qui le composent, puis à compter le nombre de pages attribuées à chaque unité éditoriale. Des comparaisons entre les différentes générations de manuels scolaires sont ensuite établies. Outre la répartition de ces unités éditoriales en nombre de pages, le contenu donne lieu à des analyses complémentaires destinées à éclairer l'évolution. Il s'agit, par exemple, de la mise en évidence des caractères des savoirs propositionnels en matière de style, de vocabulaire utilisé et de contenu informatif ou de l'analyse des types de photographies présentées. Les analyses s'appuient également sur l'introduction des manuels rédigée par les auteurs ou le directeur de collection. L'objectif de ces textes est souvent d'affirmer la conformité de l'ouvrage au programme officiel; toutefois, ils comportent parfois des informations qui permettent de mieux comprendre les intentions ou les choix didactiques des auteurs des manuels.

\section{Résultats}

\subsection{Lente transformation des manuels de terminale (élèves de 17-18 ans)}

4.1.1 Avant 1950, des manuels conçus sur le modèle des ouvrages universitaires Le manuel Delagrave, dont la première édition est parue en 1905, a pour titre Les principales puissances du monde (moins la France). C'est un ouvrage dans lequel l'écrit tient une place essentielle. Il propose des cours structurés, très détaillés. Le texte est dense et comporte de longs paragraphes, sans aucune différenciation typographique. Le plus souvent, seules les grandes parties possèdent des titres en caractère gras. Les documents sont peu nombreux. Il s'agit essentiellement de 
photographies en noir et blanc d'un format réduit mais aussi de cartes. Les tableaux statistiques et les graphiques sont rares.

Ce manuel, réédité en 1922 sous le même titre et rédigé par les mêmes auteurs, comporte 612 pages. Sur le fond, il prend en compte les bouleversements survenus en Europe après 1918; par exemple la disparition de l'empire d'Autriche-Hongrie. Sur la forme, cette édition est semblable à la précédente: $85 \%$ de l'ouvrage est constitué par des textes rédigés par les auteurs. Les documents se limitent à quelques photos en noir et blanc qui présentent des scènes intemporelles du monde rural traditionnel, des paysages, mais aussi des vues de quartiers industriels de villes européennes ou des États-Unis. On ne trouve dans cet ouvrage aucun tableau statistique, seulement quelques graphiques. Quant aux cartes, en moyenne une pour cinq pages, elles sont monochromes, souvent très chargées en raison de l'abondance des noms qui y figurent. Ce manuel ne propose aucun exercice.

L'édition de 1932 du même ouvrage est très proche de la précédente, mais elle comporte un auteur de plus, puisque Gibert est associé aux deux précédents auteurs. L'ouvrage est toujours aussi volumineux avec plus de 600 pages, et on n'y note pas d'évolution significative de la forme.

Cependant, au-delà des apparences qui sont celles d'un manuel qui expose des savoirs factuels, des nomenclatures ainsi que des localisations, l'ambition est beaucoup plus large. Dans l'avant-propos, les auteurs posent la question de ce qu'est la géographie. Comme le montre cet extrait, ils expliquent que c'est une science de synthèse qui étudie les relations entre la nature et les hommes et qui vise à développer une véritable réflexion chez les élèves:

[...] la nature et les hommes étant connus, nous les mettons aux prises. Quel parti l'homme $a-t-i l$ su tirer des conditions physiques? Dans quels cas a-t-il été contraint de subir sa tyrannie ou bien dans quelle mesure a-t-il pu s'en affranchir par son énergie, son initiative et son intelligence? La vie économique d'un État résulte, pour une bonne part, de cet ensemble d'actions et de réactions (Fallex, Mairey et Gibert, 1932, p. 7).

Il est à noter que les auteurs de cet ouvrage n'établissent aucune différence entre la science géographique et la géographie scolaire, ce qui est significatif. Le manuel fonctionne comme un ouvrage de référence, au contenu fort proche de la science géographique, à peine simplifié pour les élèves. Le travail des élèves semble consister à lire les chapitres de l'ouvrage et à en mémoriser les faits et les idées principales en complément ou comme outil d'approfondissement du cours. Ajoutons que les ouvrages étudiés qui ont été consultés au Musée aubois de l'éducation, à Troyes (Aube, France), comportent, sur certaines pages, des notes manuscrites et des phrases ou des paragraphes soulignés qui laissent penser que ces ouvrages étaient vraiment lus... et étudiés par des élèves de l'époque.

Publié en 1929 par les Éditions Hachette, le manuel de la classe terminale est rédigé par Gallouédec et Maurette et compte 611 pages. Comme les autres ouvrages publiés à l'époque, l'essentiel du volume est constitué par le texte du cours rédigé par les deux auteurs. Toutefois, cet ouvrage présente quelques spécificités. On 
trouve d'abord 13 cartes en couleurs, d'une page chacune. Ensuite, l'ouvrage comporte de nombreux graphiques en barre, dont certains occupent une page entière.

Les orientations didactiques et rédactionnelles qui attribuent une place essentielle au texte ne sont guère étonnantes, dans la mesure où, comme nous l'avons mentionné auparavant, les élèves de terminale, peu nombreux, forment une élite. En effet, les manuels de cette classe sont forts proches des ouvrages universitaires sur lesquels travailleront ces élèves lorsqu'ils seront étudiants. Ces ouvrages scolaires, outre la préparation au baccalauréat, ont de toute évidence une fonction d'initiation à l'enseignement supérieur.

\subsubsection{Entre 1950 et 1985: le triomphe du modèle de l'encyclopédie}

Dans les années 1950 et 1960, les manuels deviennent moins volumineux: le plus souvent, ils ne dépassent pas 500 pages. Si le texte occupe toujours une place primordiale, la typographie est plus aérée, et les paragraphes, plus courts. Les photographies (toujours en noir et blanc) sont beaucoup plus nombreuses, les cartes également. Les graphiques, les tableaux statistiques, les textes issus de la presse notamment, se multiplient.

\subsubsection{Un manuel qui accorde une grande place aux documents iconographiques} Édité par Belin (collection Victor Prévôt) en 1966, l'ouvrage compte 464 pages; c'est la $3^{e}$ édition, entièrement refondue, d'un ouvrage dont la première édition date de 1955. Ce manuel a eu une grande diffusion et peut être considéré comme caractéristique des ouvrages publiés à cette époque. Par certains aspects, il apparaît en rupture avec les manuels précédents. Il semble relativement proche de la production actuelle, par l'importance attribuée aux documents iconographiques, tant sur le plan quantitatif que qualitatif. À l'intérieur du manuel, les photographies monochromes sont nombreuses (on en compte 110 d'un format d'un tiers de page et parfois d'une page entière). Pourtant, ce manuel reproduit 32 photographies en couleurs, qui pour la plupart figurent sur 16 pages hors texte. Beaucoup proposent des vues de paysages, dont certaines semblent avoir surtout une fonction esthétique, comme une vue de la Boutique d'un fleuriste à Pouna (photo hors texte $\mathrm{n}^{\circ} 12$ ). Dans cet ouvrage, on peut répertorier 180 cartes, ce qui signifie en moyenne une carte pour 2,5 pages et une photo pour 3,2 pages. Les cartes n'ont plus comme objet unique la localisation, puisqu'on trouve des croquis de synthèse ou des cartes thématiques à l'échelle locale, régionale ou nationale. En dehors des cartes et des photos, ce manuel propose 18 tableaux statistiques, 12 graphiques, neuf coupes géologiques, trois schémas et deux organigrammes d'entreprises industrielles. Il faut enfin noter que certains chapitres se concluent par un court extrait de texte littéraire, centré sur un thème abordé dans le chapitre. Une autre caractéristique novatrice de ce manuel est, d'une part, de faire figurer le texte sur deux colonnes et, d'autre part, de varier les caractères typographiques. 
Malgré la présence de nombreux documents, ce manuel reste, malgré tout, un livre de textes, en l'occurrence écrit par un auteur unique, Victor Prévôt. Il est bien ancré dans la tradition des ouvrages scolaires de géographie des décennies précédentes. Ainsi, l'étude de chaque État au programme commence par l'analyse du milieu naturel, expression qui constitue le titre du premier chapitre ou de la première partie de l'étude du Royaume-Uni, des États-Unis ou de l'URSS. Le texte du cours reste assez proche, dans sa forme, de ceux des éditions des décennies précédentes, comme le prouve cet extrait:

[...] dans les pays évolués, l'homme échappe à la hantise de sa survie. Il est libéré du travail manuel et salissant, du poids d'efforts physiques sans relâche [...] Mais l'abondance des biens de consommation ne s'accompagne pas toujours d'une sagesse nécessaire à leur emploi. Les suicides, l'alcoolisme, l'enfance et l'adolescence délinquantes prolifèrent dans les pays techniquement et (apparemment) développés (Prévôt, 1955, p. 13).

\subsubsection{Un ouvrage représentatif du modèle encyclopédique}

Publié au milieu des années 1960, cet ouvrage est représentatif d'un modèle de manuel qu'on pourrait rapprocher par sa structure et par sa forme d'une encyclopédie. La richesse et la variété des illustrations et des documents proposés constituent les prémices d'une évolution qui touchera tous les manuels scolaires de géographie et qui feront écrire à Grataloup au début des années 1990, à propos des manuels scolaires de géographie: On trouve de tout, des effets de mode et des traditions, vénérables ou fossilisées, des produits de diffusion scientifique et des éléments de dialogue avec les médias grand public (Grataloup, 1992, p. 35). Les manuels de la classe terminale sont alors des ouvrages de référence, qui exposent non seulement des savoirs, mais qui veillent aussi à la clarté du discours et à la simplicité du propos, et qui possèdent une iconographie soignée. D’abord destinés aux élèves de classe terminale préparant le baccalauréat, ils visent aussi un public plus large. À cet égard, la conclusion de l'avant-propos du manuel de Prévôt est particulièrement éclairante. Elle rappelle que l'ambition de cet ouvrage est [...] d'initier efficacement les adolescents auxquels il s'adresse - et même à leurs aînés - aux mouvantes réalités humaines et économiques de notre temps (Prévôt, 1955, p. 5). Ainsi, au-delà des élèves de classe terminale, ce manuel vise aussi un public d'adultes soucieux d'avoir des informations récentes sur le monde et ses puissances. On pourrait rapprocher cette ambition d'une tradition qui s'exprimait lors de la mise en place d'un enseignement spécifique de géographie dans l'enseignement primaire et secondaire. Lefort note en effet que dans les années 1880, les auteurs de manuels de géographie [...] qui s'attèlent à la tâche de vulgarisation scolaire sont des hommes éminemment soucieux de la propagation d'un savoir auquel l'émiettement des sources fait obstacle (Lefort, 1992, p. 96).

\subsubsection{Un modèle de manuel qui domine jusqu'au milieu des années 1980}

Ce modèle de manuel, ouvrage de vulgarisation illustré, va dominer jusqu'au milieu des années 1980. Ainsi, l'ouvrage publié chez Delagrave et dirigé par Froment et 
Kienast, publié en 1983, plus de vingt ans après l'ouvrage dirigé par Victor Prévôt, et précédemment étudié, est assez proche de ce dernier. Il est abondamment illustré de photographies, mais, comme les précédents, il ne comporte aucun exercice, aucun questionnement. C'est un manuel beaucoup moins volumineux, seulement 287 pages, dans lequel le texte du cours figure sur deux colonnes. On y utilise des titres principaux de couleur bleue. Les paragraphes se différencient par un système de puces de couleur noire. Dans le texte, les mots ou les éléments de phrases clés apparaissent en caractères gras. Le texte du cours apparaît beaucoup plus froid, technique et impersonnel que celui des manuels plus anciens. Un extrait du chapitre Vers une économie mondiale de marché permet une comparaison avec les extraits de manuels plus anciens cités précédemment:

Le monde des années 30 avait vécu dans le cloisonnement: autarcie soviétique des premiers plans quinquennaux, protectionnisme peureux des économies capitalistes frappées par la crise ont montré leurs insuffisances que la SDN n'a jamais réussi à transcender. Une volonté générale de renouveau se manifeste par la Charte de San Francisco. L'ONU entend mener une politique efficace par l'intermédiaire du Conseil économique et social. Celui-ci est formé de 54 membres, élus pour trois ans par l'Assemblée générale: les cinq grands en ont toujours fait partie (Froment et Kienast, 1983, p. 14).

On peut estimer que l'évolution la plus notable des ouvrages de la classe terminale qui paraissent entre 1950 et 1985 est liée d'abord au développement de la fonction documentaire qui se manifeste par une abondance et une variété de documents, enrichis par l'usage de la couleur. Malgré tout, la fonction référentielle demeure essentielle, même si le texte du cours se trouve simplifié et réduit. En revanche, aucun exercice, aucune activité ne sont proposés dans les manuels scolaires de la classe terminale de cette génération.

\subsubsection{Les manuels d'aujourd'hui : des cahiers d'activités illustrés}

Une réédition de 1987 du manuel de terminale publié par Armand Colin semble amorcer une mutation. Cet ouvrage propose, en effet, des pages intitulées Dossiers bac. Ces dossiers n'ont pas pour objectif de mettre les élèves en activité, mais proposent des commentaires de documents corrigés correspondant à des sujets proposés lors des épreuves de géographie du baccalauréat.

Mis en application en 1989, le nouveau programme initie deux grands types de changements dans les manuels scolaires. Suivant les indications du programme, les manuels abandonnent une géographie qui étudie les puissances économiques les unes après les autres selon une logique centrée sur l'étude des relations hommesmilieux. Les logiques sociales deviennent essentielles et notamment [...] les liens qui existent entre les lieux, les acteurs et leurs activités (Clerc, 2002, p. 133). Ensuite, on peut observer un autre changement fondamental. Si les manuels de cette classe ont toujours eu comme objectif de préparer les élèves au baccalauréat, traditionnellement, le travail se répartissait implicitement entre les manuels et les professeurs. Les manuels se limitaient à la transmission de savoirs actualisés, sans aucune 
piste d'intervention dans le domaine de leur mise en œuvre pour les élèves. La proposition de sujets, l'acquisition de compétences méthodologiques nécessaires pour les traiter, c'est-à-dire rédiger une dissertation ou un commentaire de document, relevait de la responsabilité propre de l'enseignant. À partir des éditions de 1989 , tous les ouvrages sans exception comportent des exercices de préparation au baccalauréat, avec parfois des corrections et des conseils. Alors que l'évolution des manuels scolaires est généralement très lente, on observe, dans les éditions de 1989, un changement brusque qui affecte les ouvrages de tous les éditeurs. Il est vrai que la suppression au baccalauréat des sujets qui se limitent à une simple restitution de savoirs (ce qu'on appelle en France les sujets de cours) nécessite l'acquisition de compétences nouvelles chez les élèves de la classe terminale, ce qui a certainement entraîné une accélération et une unanimité dans le changement.

Pour autant, la rupture avec le modèle du manuel Encyclopédie, outil de culture générale, n'est pas totale, comme le prouve l'avant-propos de l'ouvrage édité par Bordas et rédigé par Frémont, géographe universitaire renommé. Il est donc bien normal, souligne-t-il, que les études au lycée s'achèvent par la géographie du monde contemporain. L'honnête homme de la fin du $\mathrm{xx}^{\mathrm{e}}$ siècle ne saurait l'ignorer. Le jeune bachelier doit être cet honnête homme (Frémont, 1989). Pourtant, dans le même avant-propos, l'auteur rappelle que la classe de terminale prépare au baccalauréat (p. 4). Fort de cette mention qui s'apparente à une évidence, il explique ensuite que les auteurs du manuel n'ont pas craint de montrer très pratiquement comment réaliser un croquis d'élève, une dissertation, un commentaire de documents.

Ces propos nous laissent penser qu'en 1989, entrer dans la pratique, c'est-à-dire proposer une aide méthodologique dans un ouvrage de classe terminale, est audacieux et pourrait susciter des préventions chez les enseignants prescripteurs et utilisateurs potentiels des manuels. Crainte que l'ouvrage puisse être considéré comme infantilisant pour les élèves ou crainte que les manuels scolaires empiètent sur les prérogatives et l'autonomie de l'enseignant en matière de préparation aux épreuves du baccalauréat? En réalité, ces inquiétudes s'avéreront vaines, car tous les éditeurs suivront la même voie.

Le tableau 3 présente la répartition du nombre de pages des différentes unités éditoriales pour les manuels publiés en 1989. La colonne Autres correspond aux pages consacrées à la présentation de l'ouvrage, à la table des matières et aux crédits photographiques.

Il faut noter que tous les ouvrages publiés en 1989 consacrent désormais des pages spécifiques à la préparation des épreuves du baccalauréat. Ils ne se contentent plus d'exposer des savoirs et des faits en laissant dans l'ombre les compétences nécessaires pour mémoriser, comprendre le cours et développer les habiletés nécessaires pour composer une dissertation ou pour effectuer un commentaire de documents, conformément aux épreuves du baccalauréat. En valeur absolue, le nombre de pages consacrées à des préparations de sujets varie de 14 pages $(7,5 \%)$ 
Tableau 3

Nombre de pages des différentes composantes des manuels scolaires de classe terminale publiés en 1989 (programme de 1987)

\begin{tabular}{lcccccc}
\hline Éditeur & $\begin{array}{c}\text { Pages } \\
\text { d'activités }\end{array}$ & $\begin{array}{c}\text { Pages de } \\
\text { documents }\end{array}$ & $\begin{array}{c}\text { Pages de cours } \\
\text { et documents }\end{array}$ & $\begin{array}{c}\text { Pages de cours } \\
\text { sans document }\end{array}$ & $\begin{array}{c}\text { Autres } \\
\text { Aombre total } \\
\text { de pages }\end{array}$ \\
\hline Belin & 24 & 161 & - & 129 & 6 & 320 \\
Bordas & 69 & 32 & 242 & - & 8 & 351 \\
Bréal & 14 & 3 & 292 & - & 8 & 317 \\
Hachette & 98 & 70 & 240 & - & 7 & 415 \\
Magnard & 65 & 38 & 270 & - & 11 & 384 \\
Nathan & 99 & 156 & - & 89 & 3 & 347 \\
\hline
\end{tabular}

pour le manuel publié chez Bréal, à 98 pages $(23,6 \%)$ pour le manuel publié chez Hachette, et à 99 pages $(28,5 \%)$ pour l'ouvrage publié chez Nathan. On note l'importance de l'évolution par rapport aux données qui concernent les manuels précédents. Les préparations au baccalauréat proposées adoptent des formes variées. Dans les ouvrages où ces préparations comptent plus de 60 pages, on trouve souvent des sujets traités par les auteurs de manuels, des conseils méthodologiques et des aides à la mémorisation des savoirs. Ainsi, le manuel publié chez Bordas, en 1989, comprend 69 pages de préparation méthodologique au baccalauréat, réparties en cinq rubriques. Les pages qui ont pour titre Un outil pour le bac proposent des sujets traités (16 pages). Elles sont complétées par des Approfondissements (21 pages), des Documents pour le bac (23 pages), qui sont introduits par un court texte des auteurs du manuel. Réparties dans le manuel, les cinq pages intitulées Pour aller plus loin proposent des bibliographies, des énoncés de sujets, avec quelques conseils pour les traiter. D'autres manuels adoptent des solutions plus simples dans la terminologie et ne multiplient pas les rubriques. Le manuel publié chez Belin comporte 22 pages intitulées Sujets $d u$ bac et complétées par deux pages de conseils.

Le cas de deux manuels publiés en 1989 est à souligner. Les ouvrages publiés chez Bréal et chez Magnard ont en commun de ne pas présenter des pages de préparation au baccalauréat explicitement identifiées comme telles. Pourtant, les 14 pages Dossier du premier sont en fait des commentaires de documents rédigés, conformes aux épreuves du baccalauréat. Dans ses 28 pages de Dossier thématique, le manuel publié chez Magnard procède de la même manière. De plus, les 12 pages intitulées Faisons le point proposent des listes de sujets, en traitent d'autres dans les grandes lignes, sans que le terme de préparation au baccalauréat n'apparaisse.

La présentation d'exercices dans les manuels de terminale publiés en 1989 s'inscrit dans une dynamique générale remontante. Les pages d'activités sont apparues dans les manuels de collège au milieu des années 1970, puis ont été développées dans les manuels des classes de seconde et de première une dizaine d'années plus tard, et dans les ouvrages de classe terminale à la fin des années 1980. Cette évolution s'inscrit également dans le contexte de la réforme des épreuves de géographie du baccalauréat qui se complexifient. D’abord limitées à quelques pages 
dans certains ouvrages édités en 1989, au fil des éditions et des rééditions, les activités vont prendre une place de plus en plus importante.

Le tableau 4 ci-dessous permet de répertorier le nombre de pages des différentes unités éditoriales des manuels scolaires de la classe terminale publiés en 1998 (programme de 1995). Il reprend les catégories du tableau 3. Toutefois, une catégorie supplémentaire appelée Mémorisation apparaît dans ce tableau. Elle correspond à des pages de conseils et d'exercices destinés à favoriser la mémorisation de savoirs qui figurent dans certains manuels de cette génération.

\section{Tableau 4}

Nombre de pages des différentes composantes des manuels scolaires de classe terminale publiés en 1998 (programme de 1995)

\begin{tabular}{lccccccc}
\hline Éditeur & $\begin{array}{c}\text { Pages } \\
\text { d'activités }\end{array}$ & $\begin{array}{c}\text { Pages de } \\
\text { documents }\end{array}$ & $\begin{array}{c}\text { Pages de cours } \\
\text { et documents }\end{array}$ & $\begin{array}{c}\text { Pages de cours } \\
\text { sans document }\end{array}$ & $\begin{array}{c}\text { Mémori- } \\
\text { sation }\end{array}$ & \multicolumn{2}{c}{$\begin{array}{c}\text { Nombre total } \\
\text { Autres }\end{array}$} \\
\hline Bréal & 50 & 133 & 40 & 116 & - & 21 & 360 \\
Hachette & 64 & 180 & 12 & 95 & - & 8 & 349 \\
Hatier & 75 & 152 & - & 83 & 24 & 16 & 350 \\
Magnard & 64 & 169 & 22 & 76 & 18 & 21 & 370 \\
Nathan & 96 & 131 & 8 & 80 & 11 & 9 & 335 \\
\hline
\end{tabular}

La conception générale des manuels de classe terminale publiés en 1998 se rapproche de celle des ouvrages de géographie des autres classes, puisqu'ils adoptent désormais une présentation et une structure semblables. Les chapitres s'ouvrent sur une double page comportant une ou plusieurs grandes photos, le plan du chapitre et parfois une problématique. Puis viennent des doubles pages (avec le cours sur la page de gauche et des documents sur la page de droite). Les chapitres se terminent toujours par des sujets corrigés ou comportant des conseils pour les réaliser. Entre ces différentes unités constituant les chapitres, on trouve souvent des pages consacrées à des dossiers documentaires.

Les manuels publiés en 1998 présentent des préparations de sujets du baccalauréat qui totalisent, selon les éditions, de 13,8\% (manuel publié chez Bréal) à 28,6\% (manuel publié chez Nathan) des pages de l'ouvrage. Globalement, cette proportion est supérieure à celle des manuels des éditions précédentes. Malgré leur diversité, les pages qui présentent des sujets du baccalauréat fonctionnent sur le même mode. Un sujet de l'un des trois types est proposé, une réflexion sur la problématique est généralement menée, puis des conseils sont donnés pour le traiter. Ensuite, trois cas de figure existent. Une réponse au sujet posé peut être proposée. Parfois, elle n'est que partielle et ne porte que sur une partie du sujet, et il s'agit alors pour l'élève de rédiger une réponse, guidé par des consignes plus ou moins précises. Le plus souvent, aucune correction n'est effectuée. Dans ce cas, les conseils pour rédiger la réponse sont très détaillés. Quant au manuel publié chez Hatier, il propose, selon le cas, des sujets totalement traités (la mention Corrigé figure sous l'énoncé en titre) et des sujets non traités qui portent la mention Entraînement; ils sont alors accompagnés de conseils. 
Les transformations amorcées dans les éditions de 1998 se poursuivent dans les manuels de classe terminale qui paraissent en 2004. L'ouvrage publié chez Hachette, sous la direction de Joyeux, pour un nombre de pages pratiquement égal à celui édité en 1998, ne comporte plus que 78 pages de cours, contre 95 dans la précédente édition.

Le nombre de pages consacrées aux préparations de sujets du baccalauréat reste équivalent à celui des éditions précédentes. Quant aux pages de dossiers documentaires, elles augmentent de $50 \%$. L'ouvrage publié chez Hatier, en 2004, sous la direction de Ciattoni, ne comprend plus que 47 pages de cours (contre 83 dans l'édition de 1998). Dans ce manuel, si le nombre de pages de préparation de sujets du baccalauréat reste constant, les dossiers présentant des documents nombreux et variés prennent une place essentielle, avec un nombre de pages trois fois plus important que pour le cours.

En conclusion, le tableau ci-dessous propose une synthèse de l'évolution générale de la répartition des différentes unités éditoriales au sein des manuels. Les données retenues pour chacune des périodes distinguées précédemment sont les moyennes du nombre de pages correspondant aux différentes unités éditoriales des manuels de référence cités plus haut (Tableau 2).

Tableau 5

Évolution du pourcentage du nombre de pages correspondant à chaque unité éditoriale dans les manuels scolaires de classe terminale publiés de 1905 à 2004

\begin{tabular}{lccccc}
\hline & $\begin{array}{c}\text { Pourcentage } \\
\text { du nombre } \\
\text { de pages } \\
\text { de texte du } \\
\text { cours }\end{array}$ & $\begin{array}{c}\text { Pourcentage } \\
\text { du nombre } \\
\text { de pages de texte } \\
\text { et de documents }\end{array}$ & $\begin{array}{c}\text { Pourcentage } \\
\text { du nombre de } \\
\text { pages de } \\
\text { documents }\end{array}$ & $\begin{array}{c}\text { Pourcentage } \\
\text { du nombre } \\
\text { de pages } \\
\text { d'activités }\end{array}$ & Modèle \\
\hline $\begin{array}{l}\text { Manuels } \\
\text { publiés entre } \\
1905 \text { et } 1950\end{array}$ & $60 \%$ & $25 \%$ & $15 \%$ & - & $\begin{array}{c}\text { Le manuel } \\
\text { scolaire ouvrage } \\
\text { universitaire } \\
\text { Le manuel } \\
\text { Manuels } \\
\text { publiés entre } \\
1950 \text { et 1988 }\end{array}$ \\
$\begin{array}{l}\text { Manuels } \\
\text { publiés entre } \\
1989 \text { et 2004 }\end{array}$ & $25 \%$ & $40 \%$ & $35 \%$ & & - \\
\hline
\end{tabular}

Le tableau 5 permet de mettre en évidence les dynamiques pédagogiques des manuels scolaires de géographie de la classe terminale. Une lecture verticale montre qu'en valeur relative, la diminution du nombre de pages de cours a été constante au cours de la période, alors que le nombre de pages de documents et d'activités a augmenté. Une lecture horizontale du tableau indique les caractéristiques des manuels dans chacune des trois périodes. 


\subsection{Le manuel, témoin de l'évolution du rapport aux savoirs scolaires}

4.2.1 Les quatre fonctions des manuels scolaires

Choppin (2005) attribue quatre fonctions aux manuels scolaires. La première est une fonction référentielle, appelée aussi curriculaire ou programmatique. Cet auteur la définit ainsi: [Le manuel] constitue le support privilégié de contenus éducatifs, le dépositaire de connaissances, de techniques ou de savoir-faire qu'un groupe social estime nécessaire de transmettre aux jeunes générations (2005, p. 39). La seconde fonction, dite instrumentale, concerne les méthodes d'apprentissage, les exercices et les activités proposés par les ouvrages scolaires. La troisième fonction est idéologique et culturelle. En effet, le manuel est un vecteur de transmission de l'idéologie dominante de manière explicite ou implicite. Précisons que la fonction idéologique est présente dans toutes les générations des manuels, dans ceux du passé comme dans ceux du présent, dans ceux de géographie comme dans ceux des autres disciplines. L'école et les manuels scolaires de géographie participent à la transmission de visions du monde, d'idées générales sur l'espace et son fonctionnement que la société veut transmettre aux jeunes générations. Cette fonction idéologique peut être analysée aussi bien à partir de l'étude des savoirs propositionnels, que des documents ou des exercices proposés par les manuels. Elle a donné lieu à des études spécifiques dans les manuels d'histoire français de l'enseignement secondaire (Lucas, 2001) ou dans les manuels de géographie (Niclot, 1999). Comme nous l'avons expliqué auparavant, la problématique de notre étude nous conduit à ne pas mener spécifiquement d'étude sur la fonction idéologique des manuels.

Enfin, la quatrième fonction est dite documentaire. Les manuels scolaires reproduisent un grand nombre de documents iconographiques et textuels et constituent des bases de données documentaires utilisées par les enseignants pour mettre en activité les élèves.

Pour mieux comprendre l'évolution des manuels scolaires de géographie de la classe terminale sur cette longue période, on peut établir une correspondance entre les fonctions des manuels scolaires identifiées par Choppin (2005) et les grandes unités éditoriales présentes dans les manuels. On peut en effet considérer que les pages de cours qui développent des savoirs propositionnels ont surtout une fonction référentielle ou curriculaire. Les pages d'activités (en l'occurrence, dans les manuels de terminale, les préparations au baccalauréat) ont une fonction instrumentale explicite. Enfin, les pages de documents correspondent à la fonction documentaire définie par Choppin (2005).

La typologie dégagée, qui attribue des fonctions spécifiques à chacune des trois grandes unités éditoriales, n'est ni absolue ni exclusive. Au-delà des contenus qu'il propose, le texte du savoir propositionnel possède évidemment une fonction instrumentale plus ou moins explicite. Par exemple, dans les manuels de la classe terminale, les analyses des États, qui commencent systématiquement par la description du milieu physique, orientent la manière dont l'élève restituera ses savoirs dans le cadre de la dissertation de géographie de l'épreuve du baccalauréat. Ensuite, 
il faut noter que les fonctions des manuels scolaires doivent être appréhendées dans un contexte plus global, puisqu'elles correspondent en fait aux fonctions mêmes de l'école. Si les manuels de géographie de la première moitié du $\mathrm{xx}^{\mathrm{e}}$ siècle ne proposaient aucun exercice et ne comportaient aucune référence aux méthodes d'apprentissage, cela ne signifiait évidemment pas que le professeur de géographie n'abordait pas ces questions avec ses élèves dans sa classe et que le manuel n'avait aucune fonction instrumentale. On peut penser que les auteurs de manuels ne considéraient pas que cette fonction nécessitait des développements spécifiques dans les manuels scolaires.

Jusque dans les années 1980, les exercices proposés au baccalauréat consistent essentiellement en une restitution de savoirs mémorisés. Les manuels, par leur structure (titres, enchaînements logiques), sont organisés de manière à en faciliter la mémorisation par les élèves; la fonction instrumentale est uniquement implicite. Depuis la fin des années 1980, les auteurs de manuels de géographie de la classe terminale attribuent une part importante du nombre de pages à des exercices corrigés. Les apprentissages de nature méthodologique deviennent essentiels et la fonction instrumentale est explicite. Tout naturellement, comme dans un ouvrage scolaire, le nombre de pages est limité, le choix de renforcer telle ou telle fonction se fait au détriment d'autres. On constate ainsi que le renforcement de la fonction documentaire et instrumentale dans les éditions les plus récentes se traduit par une réduction du nombre de pages consacrées à l'exposé des savoirs proprement dits (notamment du texte du cours).

\subsection{2 Évolution des fonctions des manuels et transformation du rapport au savoir}

La conclusion d'un ouvrage récent insiste notamment sur l'idée que les manuels scolaires s'insèrent dans un rapport aux savoirs scolaires et aux finalités éducatives (Lebrun, 2006, p. 35). Par rapport au savoir, nous entendons la manière dont les manuels permettent aux élèves d'accéder aux savoirs géographiques sur les États du monde, objet du programme de géographie depuis cent ans. En ce sens, le rapport au savoir est étroitement lié au statut attribué à celui-ci par la société en général, et par les élèves en particulier. Un ouvrage comportant une part importante de textes de cours qui propose des savoirs considérés comme vrais, sans que les conditions de construction de ces savoirs ou les sources mobilisées ne soient explicitées, manifeste un type de rapport particulier au savoir. Pour les élèves, ce savoir est absolu et possède un caractère de vérité. Il leur est révélé par les auteurs du manuel qui ont rédigé le texte, et par l'enseignant, qui en est le dépositaire. En revanche, un manuel dont l'essentiel du contenu est constitué de documents et d'activités fait participer les élèves au processus de construction du savoir. Celui-ci n'est donc pas donné a priori, mais résulte d'une construction, et il peut être nuancé, critiqué. Au sein des manuels les plus récents, toutefois, l'opposition n'est pas toujours aussi absolue. L'importance des pages consacrées aux documents et 
aux activités n'exclut pas l'existence de pages de cours. C'est alors la nature des liaisons établies entre les unités éditoriales qui est importante. Les savoirs propositionnels sont, dans certains cas, un texte de synthèse proposant des généralisations, mettant en contexte les savoirs construits par les activités et, dans d'autres cas, ils constituent un discours parallèle sans lien direct avec les activités, ce qui confère un double statut au savoir.

Entre longues périodes de stabilité et brusques changements, les manuels de la classe terminale ont donc beaucoup changé en cent ans. L'étude de l'évolution des fonctions des manuels scolaires qui se concrétise par l'identification des trois modèles successifs (universitaire, encyclopédique, cahier d'activités) montre que la nature et le statut du savoir géographique scolaire se sont profondément transformés au cours du siècle dernier. La compréhension de ces changements passe par leur mise en relation avec l'évolution du savoir scolaire en géographie et les transformations du rapport qu'entretiennent les élèves et les enseignants avec ce savoir.

Un premier type de rapport au savoir domine jusque vers les années 1950. Dans celui-ci, le savoir géographique est extérieur à l'élève, tout entier détenu par les auteurs de manuels. Comme le remarque Clerc (2002, p. 159), La culture scolaire en géographie est fondée sur la transmission de savoirs factuels relatifs à des morceaux de l'espace terrestre. Les quelques documents qui figurent dans les ouvrages scolaires ont surtout une fonction de légitimation ou d'aide à la compréhension. Dans les éditions postérieures, un deuxième type de rapport au savoir peut être mis en évidence. Le cours conserve certes une place essentielle, mais la multiplication des documents de tous ordres remet en cause l'extériorité et le caractère absolu du savoir géographique scolaire. Les documents nuancent, confirment ou fondent le texte du cours qui perd ainsi sa suprématie et son caractère de vérité. Dans les manuels actuels, les documents vont même, parfois, jusqu'à se substituer au cours. C'est le cas des nombreux dossiers thématiques qui réunissent un ensemble de documents autour d'un thème central dont l'objectif est de permettre à l'élève de construire ses propres savoirs sur un sujet donné, sans que la question ne soit traitée sous forme de cours. C'est donc un troisième type de rapport au savoir qui apparaît, dans lequel le savoir géographique peut donner lieu à des constructions personnelles et devient ainsi relatif et contingent.

En relation avec les évolutions du savoir scolaire, la question du rapport au savoir des élèves est contenue dans ces interrogations formulées par Charlot à propos des jeunes issus des milieux populaires qui fréquentent les lycées professionnels de banlieue: Quel est le rapport à l'école, au savoir, et plus généralement à «l'apprendre», d'un jeune de milieu populaire, en France, aujourd'hui? Que signifient pour lui apprendre et savoir? Quel sens cela présente-t-il pour lui d'aller à l'école et d'y travailler - ou de ne pas y travailler? (Charlot, 1999, p. 329). Ces questions, qui se réfèrent aux élèves de lycée professionnel, peuvent également se poser pour les élèves de collège et de lycée d'enseignement général. Bautier et Rochex (1998), sur la question du rapport au savoir, distinguent deux types d'élèves: les héritiers et 
les nouveaux lycéens. Les premiers, issus de milieux culturellement favorisés, comprennent les enjeux des savoirs et des disciplines scolaires. Quant aux seconds, aux origines sociales plus populaires, ils appuient leur réflexion sur des valeurs (le bien, le mal) qui permettent de donner des réponses à toutes les questions qui se posent. Pour eux, L'énoncé-type est un énoncé injonctif-normatif (il faut mettre en ouvre une norme) présentant un stéréotype, en général moral, qui dit les valeurs du groupe qui ne sont ni mises en débat ni interrogées (Bautier et Rochex, 1998, p. 91). Ces deux auteurs montrent aussi que ces élèves ont des difficultés à argumenter ou à généraliser à partir d'un cas particulier.

Les transformations des manuels scolaires de la classe terminale peuvent être interprétées en référence à l'évolution du rapport au savoir des élèves, elle-même liée à ce qu'il est convenu d'appeler le phénomène de massification de l'enseignement. Conçus sur le modèle des ouvrages universitaires, les manuels de la première moitié du $\mathrm{xx}^{\mathrm{e}}$ siècle sont bien adaptés à un public d'élèves habitués à lire, maîtrisant parfaitement l'écrit et pour lesquels le sens des savoirs scolaires s'impose avec le poids de l'évidence. Le manuel conçu sur le modèle de l'encyclopédie correspond, quant à lui, à la période d'augmentation régulière du nombre d'élèves poursuivant des études en lycée qui s'observe à partir de la fin des années 1950, caractérisée par un accès beaucoup plus important des enfants des classes moyennes. Aussi, comme nous l'avons vu précédemment, dans les manuels publiés entre 1950 et 1985, le texte du cours se simplifie, les documents se multiplient. Pourtant, les manuels continuent de privilégier le texte du cours. Au fond, le manuel de type encyclopédie n'est qu'une variante des ouvrages de la génération précédente. La mémorisation de savoirs sur les États du monde reste l'objectif premier de la géographie scolaire de la classe terminale et des épreuves de géographie du baccalauréat. Les auteurs ne font qu'adapter le contenu et la présentation des manuels aux évolutions du public scolaire moins familier de l'écrit, plus sensible à l'image et spectateur assidu de médias audiovisuels. La rupture qui s'observe dans les manuels publiés en 1989 correspond, avec quelques années de décalage seulement, à l'arrivée en classe terminale des premiers élèves formés dans le collège unique à partir de 1977. Le mouvement de massification a modifié considérablement la composition des classes de lycée, avec l'apparition de nouveaux lycéens. Alors que, pour les générations précédentes, la rédaction d'une dissertation ou d'un commentaire de documents ne nécessitait pas d'exercices d'entraînement particuliers en classe terminale, les manuels de la génération actuelle en proposent. Les auteurs des ouvrages insistent aussi sur les techniques de mémorisation. Ils ne se contentent plus de présenter des savoirs, ils proposent également des activités destinées à faire acquérir aux élèves les compétences nécessaires pour réussir l'épreuve de géographie du baccalauréat.

Finalement, l'évolution des manuels de terminale est révélatrice des transformations du rapport au savoir à enseigner qu'ont les enseignants de lycée. De manière directe, on pourrait dire qu'aujourd'hui, enseigner la géographie ce n'est 
plus uniquement donner aux élèves des informations sur les relations entre les hommes et le milieu ou livrer des éléments de compréhension sur le fonctionnement de l'espace terrestre et des territoires, comme cela a été longtemps le cas. La place accordée aux activités dans les manuels de collège et de lycée va dans le sens de l'évolution générale de cette discipline scolaire qui valorise la construction des savoirs par les élèves eux-mêmes, ce qui passe par l'acquisition de compétences nécessaires pour y parvenir. En s'appuyant notamment sur des documents reproduits dans les manuels et sur les exercices qui y figurent, les enseignants ont pris l'habitude de travailler avec leurs élèves et de mettre en pratique ce que les programmes officiels appellent la pédagogie du document (Niclot et Aroq, 2006). Selon la logique développée dans les manuels de géographie de collège et de lycée, les enseignants d'aujourd'hui forment les élèves à construire leurs savoirs, à mettre en œuvre des savoir-faire et tentent de développer chez eux une réflexion autonome. On comprendrait donc mal que les manuels de géographie de la classe terminale ne soient pas dans la continuité des ouvrages des autres niveaux.

\section{Conclusion}

Une analyse des manuels de géographie de la classe terminale publiés pendant un siècle montre que les ouvrages actuels ont très peu de points communs avec ceux du passé. L'étude de la forme et du contenu des manuels de cette classe a permis d'identifier quelles transformations en profondeur les ont affectés. Au fil des éditions successives, la fonction curriculaire s'est atténuée au profit de la fonction documentaire et, plus récemment, de la fonction instrumentale. Au manuel conçu sur le modèle des ouvrages universitaires, comportant essentiellement un cours rédigé par ses auteurs, a succédé le manuel réalisé sur le modèle de l'encyclopédie. Aujourd'hui, les ouvrages scolaires ne sont plus des livres de texte, leur structure est éclatée entre pages de cours, de documents divers, d'apprentissages méthodologiques, de préparation aux sujets d'examens. Ces transformations peuvent être mises en relation avec les évolutions du public scolaire accédant à la classe terminale, caractérisé par une augmentation rapide depuis 25 ans et, par voie de conséquences, par une hétérogénéité accrue. Les manuels se sont adaptés aux nouvelles formes de rapport au savoir qu'entretiennent les élèves et les enseignants de cette discipline. Ils ont intégré les transformations épistémologiques du savoir géographique savant et se sont conformés aux orientations pédagogiques et didactiques définies par les programmes qui se sont succédé. Ils ont pris en compte les transformations des modalités de l'épreuve de géographie du baccalauréat. Enfin, les manuels de terminale ont adopté les mêmes normes éditoriales que les manuels des autres niveaux. Ils ont été les derniers à le faire, mettant fin aux spécificités qui ont longtemps marqué les manuels de géographie de la classe terminale. L'étude des manuels scolaires sur une longue période montre l'extrême complexité et l'enchevêtrement des éléments qui déterminent l'évolution générale des ouvrages scolaires. 
Le travail présenté ici vise à caractériser l'évolution formelle des manuels de géographie de la classe terminale, en étudiant la fonction pédagogique des pages proposées et leur importance relative dans chaque génération d'ouvrages publiés en France depuis un siècle. Différents modèles de manuels ont été identifiés et, à partir de ces analyses, l'évolution du rapport au savoir des élèves et des professeurs a été inférée. Les résultats obtenus, qui ne concernent qu'un seul niveau de classe, la terminale, devront être confirmés par des études portant sur des manuels de géographie d'autres classes. Il en est de même pour la méthodologie utilisée, qui rompt avec les traditionnelles analyses de contenu privilégiées par la plupart des études sur les manuels scolaires. Cette nouvelle approche appliquée à des manuels de géographie pourrait certainement être utilisée pour les manuels d'autres disciplines scolaires.

ENGLISH TITLE 100 years of evolution of geography textbooks for year 12 students in France: deep transformations in the relationship to school knowledge in geography

SUMmary - This study is an analysis of geography textbooks for year 12 students in France over a period of 100 years and focuses on their pedagogical evolution. It is based on the analysis of twenty-one textbooks published from 1905 to 2006. It aims to identify the main pedagogical models to which they belong. On the one hand, changes in textbooks are linked to the evolution of the French education system and on the other hand, to the evolution of students' and teachers' relationship to school knowledge in geography.

KEY WORDS • geography, France, textbook, relationship to school knowledge, pedagogical patterns.

Título en ESPAÑol - Cien años de evolución de los libros de texto de geografía para alumnos de $5^{\circ}$ de secundaria en Francia : las profundas transformaciones de la relación con los saberes escolares en geografía

RESUMEN • Este estudio analiza los libros de geografía franceses para alumnos de 50 de secundaria en une periodo de cien años y estudia su evolución pedagógica. Se basa en el análisis de 21 libros de texto publicados entre 1905 y 2006. Su objetivo es identificar a los principales modelos pedagógicos a los cuales se refieren. Se relacionan las transformaciones de los libros de texto con la evolución del sistema educativo francés, por una parte, y con la evolución de la relación que tienen los alumnos y los docentes con los saberes en geografía, por otra parte.

PALABRAS CLAVES - geografía, Francia, libro de texto, relación con el saber, modelo pedagógico.

\section{Références}

Bautier, E. et Rochex, J.-Y. (1998). L'expérience scolaire des nouveaux lycéens. Démocratisation ou massification? Paris, France: Armand Colin.

Charlot, B. (1999). Le rapport au savoir en milieu populaire. Une recherche dans les lycées professionnels de banlieue. Paris, France: Anthropos.

Chevalier, J.-P. (2002). Images de l'Europe dans les manuels de géographie français. Travaux de l'Institut de géographie de Reims, 110, 33-49. 
Choppin, A. (2005). L'édition scolaire française et ses contraintes: une perspective historique. Dans E. Bruillard (Dir.) : Manuels scolaires, regards croisés. Caen, France: Centre régional de documentation pédagogique de Basse-Normandie.

Clerc, P. (2002). La culture scolaire en géographie. Le monde dans la classe. Rennes, France: Presses universitaires de Rennes.

De la Borderie, R. (1997). Éducation à l'image et aux médias. Paris, France: Nathan Pédagogie.

Delbos, G. et Joron, P. (1984). La transmission des savoirs. Paris, France: Maison des Sciences de l'Homme.

De Rosnay, J. (1975). Le Macroscope, vers une vision globale. Paris, France: Le Seuil.

Desiré, P.-E. et Pagney, P. (1996). De la géographie des manuels à la géographie vécue: un douloureux décalage. Historiens et géographes, 352, 85-90.

Freycinet-Deminjon, J. (1969). Les manuels d'histoire de l'école libre 1862 - 1959. De la loi Ferry à la loi Debré. Paris, France: Gelin.

Grataloup, C. (1992). Les moyens de l'imaginaire du géographe: modes de représentation du monde et de la discipline. Dans F. Audigier (Dir.): Actes du septième colloque des didactiques de l'histoire, de la géographie, des sciences sociales. Paris, France: Institut national de recherche pédagogique.

Inspection générale de l'Éducation nationale (1998). Le manuel scolaire. Programme de travail 1997-1998. Paris, France: La Documentation française.

Lebrun, J. (2006). Les manuels scolaires «réformés» au primaire: quelle place pour la médiation de l'enseignant et les apprentissages des élèves? Dans J. Lebrun, J. Bédard, A. Hasni et V. Grenon (Dir.): Le matériel didactique et pédagogique: soutien à l'appropriation ou déterminant de l'intervention éducative. Laval, Québec: Presses de l'Université Laval.

Lefort, I. (1992). La lettre et l'esprit, Géographie scolaire et Géographie savante en France 1870-1970. Mémoires et documents de géographie. Paris, France: Centre national de la recherche scientifique.

Le Fustec, A. et Sivan P. (2006). Les manuels de français au feu des critiques. Dans E. Bruillard (Dir.): Manuels scolaires, regards croisés. Caen, France: Centre régional de documentation pédagogique de Basse-Normandie.

Lenoir, Y. (2006). Orientations épistémologiques du nouveau curriculum et contribution des manuels scolaires à leur mise en œuvre. Dans J. Lebrun, J. Bédard, A. Hasni et V. Grenon (Dir.): Le matériel didactique et pédagogique: soutien à l'appropriation ou déterminant de l'intervention éducative. Québec, Québec: Presses de l'Université Laval.

Lucas, N. (2001). Enseigner l'histoire dans le secondaire. Manuels et enseignement depuis 1902. Rennes, France: Presses universitaires de Rennes.

Mendibil, D. (1997). Textes et images de l'iconographie de la France (de 1840 à 1990). Essai d'iconographie géographique. Thèse de doctorat inédite, Université de Paris I - Panthéon Sorbonne, Paris, France.

Ministère de l'Éducation nationale (1997). Nouvelles épreuves d'histoire et de géographie du baccalauréat pour la session de 1999. Bulletin officiel de l'éducation nationale (spécial 2). Paris, France: Imprimerie Nationale. 
Niclot, D. et Aroq, C. (2006). Les évolutions récentes des manuels de géographie de l'enseignement secondaire français et les pratiques déclarées des enseignants français. Dans J. Lebrun, J. Bédard, A. Hasni et V. Grenon (Dir.) : Le matériel didactique et pédagogique: soutien à l'appropriation ou déterminant de l'intervention éducative. Québec, Québec: Presses de l'Université Laval.

Niclot, D. (2001). Les manuels de géographie et la géographie des manuels. Travaux de l'Institut de Géographie de Reims, 109, 104 -131.

Niclot, D. (1999). Images de la vulgate scolaire dans les manuels de géographie français de la classe de seconde, publiés de 1981 à 1996. Cahiers de géographie du Québec, 43(120), 605-624.

Sourp, R. (2002). Les mutations de la causalité dans le raisonnement scolaire: l'exemple de la présentation de l'Europe en classe de quatrième. L'information géographique, 65, 244-261.

Monsieur Daniel Niclot est chercheur au Laboratoire d'étude et de recherche sur les professionnalisations (LERP)

\section{Correspondance}

daniel.niclot@univ-reims.fr

Ce texte a été révisé par Isabelle Gauvin.

Texte reçu le: 8 mars 2007

Version finale reçue le: 15 mai 2008

Accepté le: 3 juillet 2008 